\title{
Epidémiologie Des Anomalies Morphologiques Néonatales En Milieu Hospitalier À Kara (Nord Togo) De 2014 À 2019
}

\author{
Segbedji KAR, \\ Tchagbele $\mathrm{O}-\mathrm{B}$,
}

Département de pédiatrie, CHU Kara, Université de Kara, Togo

$$
\text { Takassi OE, }
$$

Département de pédiatrie, CHU- SO, Université de Lomé, Togo

\section{Kombieni $K$,}

Talboussouma SM,

Agrigna H,

Département de pédiatrie, CHU Kara, Université de Kara, Togo

Djadou KE,

Département de pédiatrie, CHU- SO, Université de Lomé, Togo

Gbadoé AD,

Azoumah KD

Département de pédiatrie, CHU Kara, Université de Kara, Togo

\section{Doi:10.19044/esj.2022.v18n3p1}

Submitted: 17 December 2021

Accepted: 03 January 2022

Published: 31 January 2022
Copyright 2022 Author(s)

Under Creative Commons BY-NC-ND 4.0 OPEN ACCESS

Cite As:

Segbedji KAR, Tchagbele O-B, Takassi OE, Kombieni K, Talboussouma SM, Agrigna H, Atakouma YD, Djadou KE, Gbadoé AD,\& Azoumah KD.,(2022). Epidémiologie Des Anomalies Morphologiques Néonatales En Milieu Hospitalier À Kara (Nord Togo) De 2014 À 2019 European Scientific Journal, ESJ, 18 (3), 1.

https://doi.org/10.19044/esj.2022.v18n3p1

\section{Résumé}

La rareté apparente des malformations néonatales cliniquement décelables au Nord Togo a motivé la présente étude de série de cas visant à décrire l'aspect épidémiologique de ces malformations en milieu hospitalier. La prévalence des malformations néonatales était de de 3,6 pour 1000 naissance vivantes. Les malformations retrouvées concernaient essentiellement la tête et la face (37,2\%), les membres (33\%) et la colonne vertébrale (14,9\%), les organes génitaux externes (9,4\%) et l'abdomen (6,3\%). Les nouveau-nés malformés étaient issus pour la plupart des mères âgées entre 26 et 30 ans (31,25\%), ménagères $(67,2 \%)$, et de niveau scolaire primaire 
(47\%). Les facteurs de risque susceptibles d'être responsables de la survenue des malformations congénitales sont l'âge maternel avancé, le bas niveau socio-économique, l’alcoolisme, et la multiparité (43,7\%).

Mots clés: Malformation, Congénitale, Épidémiologie, Facteur De Risque, Prévalence, Togo

\title{
Epidemiology of Morphological Birth Defects in Hospital Ward in Kara From 2014 To 2019
}

\author{
Segbedji KAR, \\ Tchagbele $\mathrm{O}-\mathrm{B}$, \\ Département de pédiatrie, CHU Kara, Université de Kara, Togo \\ Takassi OE, \\ Département de pédiatrie, CHU- SO, Université de Lomé, Togo \\ Kombieni K, \\ Talboussouma SM, \\ Agrigna H, \\ Département de pédiatrie, CHU Kara, Université de Kara, Togo \\ Djadou KE, \\ Département de pédiatrie, CHU- SO, Université de Lomé, Togo \\ Gbadoé AD, \\ Azoumah KD \\ Département de pédiatrie, CHU Kara, Université de Kara, Togo
}

\section{Abstract}

The apparent rarity of clinically detectable neonatal malformations in North Togo motivated the present case series study aimed at describing the epidemiology of these malformations in hospital settings. The prevalence of neonatal malformations was 3.6 per 1000 live births. The malformations found mainly concerned the head and face (37.2\%), limbs (33\%) and spine (14.9\%), external genitalia (9.4\%) and abdomen (6.3\%). The malformed newborns were mostly from mothers aged between 26 and 30 years (31.25\%), housewives (67.2\%), and primary school level (47\%). The risk factors likely to be responsible for the occurrence of congenital malformations are maternal advanced age, low socioeconomic status, alcoholism, and multiparity (43.7\%).

Keywords: Malformation, Congenital, Epidemiology, Risk Factor, Prevalence, Togo 


\section{Introduction}

Les malformations congénitales sont la conséquence de plusieurs facteurs génétiques, infectieux, médicamenteux ou environnementaux mais dont le mécanisme n’est pas encore élucidé à ce jour.

En Europe, la prévalence des malformations congénitales est d'environ 2,5\% avec une létalité néonatale comprise entre 20 et 25\% (OMS, 2010).

Dans les pays à ressources limitées, les politiques de santé publique s’orientent vers les trois principales causes de mortalité néonatale (asphyxie périnatale, prématurité et infection néonatale) bien que les malformations congénitales soient également une cause de décès néonatale (Sabiri et al., 2013). Ainsi, peu de données sur la prévalence des malformations néonatales sont disponibles. La prévalence des malformations varient de 2,9 à 9 pour 1000 naissances vivantes selon les études hospitalières souvent peu exhaustives (Sabiri et al., 2013 ; Youl, 2011 ; Lubala, 2011 ; Assimady et al., 1997).

Les malformations congénitales, en plus d'être vécues comme un cauchemar compte tenu des difficultés de prise en charge, sont considérées comme des faits mystiques et religieux en Afrique (Lubala, 2011).

La présente étude visait à décrire le profil épidémiologique des malformations néonatales cliniquement décelables au CHU Kara (Togo). De façon spécifique, l'étude avait pour objectifs de déterminer la prévalence des malformations, d'identifier leurs principaux facteurs de risque et de décrire leur prise en charge en milieu sous-équipé et en équipe pluridisciplinaire.

\section{Patients et méthodes}

Les services de pédiatries et de gynéco-obstétriques de référence publique de la ville de Kara à savoir le Centre Hospitalier Universitaire (CHU) et le Centre Hospitalier Régional (CHR-Tomdè) nous ont servi de cadre d'étude.

Il s’est agi d'une étude de série de cas colligés de janvier 2014 à février 2019 dans les deux principaux centres hospitaliers de la ville de Kara (Nord Togo). L’étude a porté sur tous les nouveau-nés (mort-nés ou nés vivants) présentant une malformation congénitale cliniquement visible à la maternité et/ou en hospitalisation pédiatrique.

L’outil de collecte de données utilisé était une fiche d'enquête structurée en trois parties. La première partie a concerné les variables sociodémographiques des mères, la deuxième partie les antécédents de la mère et la troisième partie les variables sur le nouveau-né. L’administration indirecte par un personnel soignant de questionnaire, le dépouillement des registres d'accouchement et des observations, et des dossiers médicaux des nouveau-nés ont été les techniques de collecte utilisées. L’anonymat des enfants malformés et sur le consentement éclairé de leurs mères étaient respectés lors de la collecte des données. 
L'analyse et le traitement des données ont été faits à l'aide du logiciel Excel 2007 et Word 2013.

\section{Résultats}

\section{Prévalence des malformations}

Pendant la période d'étude, nous avons enregistré 64 cas de malformations sur un effectif de 13363 accouchements, soit une prévalence de 3,6 pour 1000 naissances.

\section{Différents types de malformations}

$\mathrm{Au}$ total nous avons identifié 19 types de malformations que présentaient les nouveau-nés vivants et les mort-nés. Les plus fréquentes étaient : les pieds bots et ou équins (21,9\%), l’hydrocéphalie $(15,6 \%)$ et le spina-bifida (12,5\%). Les différents types de malformations sont présentés dans le tableau I.

Tableau I : Répartition des différents types de malformations en fonction de leur localisation

\begin{tabular}{ccc}
\hline & Effectif & Pourcentage (\%) \\
\hline Tête et cou & $\mathbf{2 5}$ & $\mathbf{3 9}$ \\
Hydrocéphalie & 11 & 17,2 \\
Anencéphalie & 5 & 7,8 \\
Fente palatine & 5 & 7,8 \\
Myéloencéphalocèle & 2 & 3,1 \\
Microcéphalie & 2 & 3,1 \\
Membres & $\mathbf{2 0}$ & $\mathbf{3 1 , 3}$ \\
Pieds bots et ou équins & 14 & 21,9 \\
Polydactylie ou syndactylie & 6 & 9,4 \\
Colonne vertébrale & $\mathbf{9}$ & $\mathbf{1 4}$ \\
Spina-bifida & 9 & 14,0 \\
Paroi abdominale & $\mathbf{4}$ & $\mathbf{6 , 3}$ \\
Laparoschisis & 3 & 4,7 \\
Omphalocèle & 1 & 1,6 \\
Hypospadias & $\mathbf{6}$ & $\mathbf{9 , 4}$ \\
Organes génitaux externes & 5 & 7,8 \\
Aphalie (absence complète de & 1 & 1,6 \\
sexe) & & \\
\hline
\end{tabular}

\section{Variables sociodémographiques des mères}

L'âge moyen des mères des enfants malformés était de 31 ans avec des extrêmes de 16 et 41 ans. Deux mères (3,2\%) étaient des adolescentes et dixhuit mères avaient un âge supérieur à 38 ans.

Les mères avaient un niveau scolaire primaire (47\%), secondaire (30\%), mais des analphabètes dans $23 \%$ des cas. 
Concernant leurs occupations, les mères étaient des ménagères (67,2\%), des commerçantes $(23,4 \%)$, des fonctionnaires $(6,3 \%)$ et des étudiantes (3,1\%).

Les nouveau-nés étaient de sexe féminin (37\%) ou masculin (61\%). Le sexe était absent dans un cas.

\section{Facteurs de risques maternels}

Les mères ne souffrant d'aucune maladie chronique représentaient 90,6\% des cas. Les antécédents pathologiques (9,4\% des cas) étaient dominés par la co-infection hépatite $B$ et l'infection au virus de l'immunodéficience humaine (3 cas), l'hépatite virale $B$ isolée (2 cas), le diabète (1 cas), l'hypertension artérielle (1 cas).

Les multigestes représentaient $42,2 \%$ et 9,4\% étaient des grandes multigestes. Les primigestes et les paucigestes représantaient respectivement $25 \%$ et $23,4 \%$ des cas.

Les multipares représentaient $43,7 \%$ et $6,3 \%$ étaient des grandes multipares. Les primipares et les paucipares représentaient respectivement $24 \%$ et $26 \%$ des cas.

Les nouveau-nés issus d'un accouchement par voie basse étaient de 41 soit $64,1 \%$ des cas et 23 étaient issus d'un accouchement par la voie haute (césarienne) soit 35,9\% des cas.

Les mères qui consommaient de l'alcool recensées dans cette étude représentaient $17,2 \%$ des cas.

\section{Variables concernant les nouveau-nés}

La grossesse était menée à terme dans 56 cas (87,5\%) et la prématurité a été notée dans 8 (12,5\%).

Vingt-trois nouveau-nés étaient réanimés soit 35,9\% des cas et 41 non réanimés soit $64,1 \%$ des cas. Les nouveau-nés vivants étaient 52 (81,2\%) et 12 mort-nés (18,8\%).

La taille moyenne à la naissance était $43,5 \mathrm{~cm}$ avec des extrêmes de $36 \mathrm{~cm}$ et $51 \mathrm{~cm}$. Le périmètre crânien moyen à la naissance était de $35,5 \mathrm{~cm}$ avec des extrêmes de $20 \mathrm{~cm}$ et $51 \mathrm{~cm}$. Le poids moyen à la naissance était de 2700 grammes aves des extrêmes de 1100 grammes et 4300 grammes.

\section{Prise en charge}

Dans 43,8\% des cas, les nouveau-nés admis en pédiatrie nécessitaient une intervention chirurgicale (tableau II).

Tableau II : Répartition des cas de malformations en fonction de la décision médicale

\begin{tabular}{ccc}
\hline & Effectif & Pourcentage (\%) \\
\hline Intervention chirurgicale & 28 & 43,8 \\
Aucune décision médicale nécessaire & 12 & 18,8
\end{tabular}




\begin{tabular}{ccc} 
Immobilisation plâtrée et rééducation & 12 & 18,8 \\
Non précisée & 10 & 15,6 \\
Ligature pour polydactylie & 1 & 1,6 \\
Immobilisation plâtrée, rééducation et & 1 & 1,6 \\
badigeonnage & & $\mathbf{1 0 0}$ \\
\hline Total & $\mathbf{6 4}$ & \\
\hline
\end{tabular}

\section{Discussion \\ Prévalence}

La prévalence des malformations congénitales cliniquement décelables en milieu hospitalier à Kara était de 3,6 pour 1000 naissances. Cette prévalence est inférieure à celle de Kamla et al (2017) dans leur étude sur l'épidémiologie des malformations congénitales visibles à la naissance à Yaoundé en 2017 qui trouvaient une prévalence de 9 cas pour 1000 naissances vivantes dans 10 maternités. Elle est largement inférieure à celui de Lubala (2011) à Lubumbashi. La variabilité de ces résultats s'expliquerait par les méthodes utilisées et la durée des études.

Dans notre étude, il y avait une prédominance des malformations de la tête et de la face (37,2\%) suivies des malformations des membres (33\%) et de la colonne vertébrale $(14,9 \%)$. Les anomalies du crâne $(33,5 \%)$ et de la face (29,5\%) étaient prédominantes (Coulibaly et al., 1997). Cependant, les malformations de membres étaient les plus fréquentes dans 63,93\% des cas à Cotonou (Fiogbe et al., 2012) et dans (35,4\%) à Ouagadougou (Youl, 2011). Il y avait une prédominance masculine dans notre travail, ce qui est similaire aux résultats rapportés à Abidjan en milieu hospitalier (Mouafo et al., 2004). Ce résultat pourrait s'expliquer par le fait qu'on accouchait plus le sexe masculin dans les centres étudiés. D’autres auteurs rapportent au contraire une prédominance féminine (Konaté, 2008 ; Youl, 2011).

Un nouveau-né sans organe génital externe (aphalie) a été retrouvé dans cette étude. Kamla et al. (2017) ont retrouvé quatre nouveau-nés polymalformés sans organes génitaux externes. Cette prédominance étant variable, nous ne pouvons pas tirer de conclusion entre le sexe et la survenue de malformations congénitales. Néanmoins certaines malformations sont spécifiques au sexe masculin (hypospadias et kyste du cordon).

\section{Facteurs de risque}

L'âge moyen des mères était de 31 ans, la tranche d'âge comprise entre 26 et 30 ans (31,3\%) a été la plus concernée. Ces résultats sont proches de ceux retrouvés à Rabat au Maroc (Benzerouale, 2017) où l'âge moyen des mères était de 29,5 ans et un pic constaté pour la tranche d'âge de 20-35 ans. Ce résultat pourrait s'expliquer par le fait que cette tranche d'âge correspond à l'âge moyen de la procréation. Le risque de malformation est élevé après 
l'âge de 38 ans (Shawky et al., 2011). En effet, les études génétiques montrent bien que l'âge maternel avancé est un facteur déterminant dans l'apparition d'enfants porteurs d'anomalies génétiques et par conséquent de malformations congénitales (Hollier et al., 2000 ; Nazer et al., 2007).

Dans $67,2 \%$ cas, les nouveau-nés étaient issus d'une mère ménagère. Youl et Alassane ont fait le même constat en trouvant respectivement 92,5\% et 83,7\% (Youl, 2011 ; Alassane,2006). La non adhésion des femmes à la supplémentation systématique en fer acide folique et le mauvais suivi de la grossesse pourraient expliquer certaines malformations à l'instar du spina bifida. Le niveau socio-économique représente un facteur de risque puisqu'il est responsable dans la majorité des cas des malformations du tube neural ainsi que des fentes palatines (Benzerouale, 2017). Un bas niveau socioéconomique est souvent corrélé à un mauvais suivi de la grossesse et à une insuffisance dans la prévention des malformations.

Le risque de malformations était réduit chez les primipares (12\%) et s'accroissait chez les multipares (43,7\%). Youl a fait le même constat en trouvant une fréquence de 51,5\% et expliquait que le risque de malformations congénitales augmentait avec la parité de façon significative (Youl, 2011). Par contre Konaté (Konate, 2008) avait trouvé que les malformations congénitales étaient plus fréquentes chez les primipares.

Cinq mères étaient suivies pour des pathologies chroniques (7,8\%). Le diabète maternel, l'asthme et d'autres affections chroniques ont été incriminés dans la survenue de malformations congénitales (Sabiri et al., 2013 ; Agence de la santé publique du Canada, 2013 ; Benzerouale, 2017). Les maladies chroniques peuvent expliquer l'apparition des malformations congénitales dans notre étude, c'est le cas de l'hypertension artérielle, l'hépatite B, le diabète, l'asthme et le VIH/SIDA.

Dans cette étude, les mères consommatrices d'alcool pendant leur grossesse étaient de 17,2\%. Cette fréquence est supérieure aux 12,5\% retrouvés à Lubumbashi (Lubala, 2011). Les effets de l'éthanol sur le fœtus peuvent se classer en 4 catégories : retard de croissance, anomalies neurologiques, dysmorphies faciales et autres malformations (Gillerot, 2005). La prévention du spina bifida se fait par une prise périconceptionnelle régulière de l'acide folique (Gillerot, 2011) chez les femmes à risque de carence en vitamine B9. Ce médicament est disponible à moindre coût dans notre pays.

\section{Conclusion}

Les malformations congénitales sont méconnues par la population et sous évaluées à Kara. Elles concernent surtout les pieds bots, l'hydrocéphalie, le spina bifida et les polydactylies. Les facteurs de risque incriminés dans la survenue des malformations congénitales étaient: le niveau socio-économique 
bas, la multiparité, et l'alcoolisme au cours de la grossesse. Certaines de ces malformations engagent le pronostic vital et surtout fonctionnel des nouveaunés

\section{References:}

1. Agence de la santé publique du Canada (2013). Les anomalies congénitales au Canada: rapport de la surveillance sur la santé périnatale. Ottawa, septembre 2013.

2. Alassane, S. (2006). Etude des malformations congénitales dans le service de pédiatrie de l'hôpital Gabriel TOURE à propos de 98 cas. Bamako, Thèse, Médecine, 73P.

3. Assimady, K, Atakouma, DY, Goujard, J, Dormons, S, Du Ong, Q, Hodonou, K, Ategbo, S, Schmitt Ehry G. (1977). Enquête épidémiologique sur les malformations congénitales à Lomé. Journée APANF, abstrat, 4-6, p.35.

4. Benzerouale, O. Les anomalies morphologiques du nouveau-né. Etude rétrospective à propos de 68 cas colligés au niveau du centre hospitalier provincial de kenitra. (2017). Thèse médecine, Rabat $\mathrm{N}^{\circ} 98$, 120p. [En ligne] https://docplayer.fr/81324317-Les-anomaliesmorphologiques-du-nouveau-ne-etude-retrospective-a-propos-de-68cas-colliges-au-niveau-du-centre-hospitalier-provincial-dekenitra.html

5. Coulibaly-Zerbo, F, Amorissani-Folquet, M, Kacou-Kacou, A, Sylla, M. (1997). Etude épidémiologique des malformations congénitales. Médecine d'Afrique Noire, 44 : 409-414.

6. Fiogbe, MA, Goudjo, E, Gbenou, AS, Fiogbe, DA, Tonato-Bagnan, AJ. (2013). Les malformations congénitales cliniquement visibles et facteurs de risque répertoriés chez les nouveau-nés à Cotonou. J Rech Sci Univ Lomé, 15 (1): 67-74.

7. Gillerot, Y, Dan, B. (2005). Le syndrome d'alcoolisme fœtal. Education du patient et enjeux de santé, 23 (3): 70-72.

8. Gillerot, Y. (2011). Prévention du spina bifida et d'autres malformations par l'acide folique. IPG-Centre de Génétique Humaine de Loverval, 41, 1-3.

9. Kamla, JI, Kamgaing N, Nguifo Fongang, EJ3, Fondop, J, Billong, S, Djientcheu, VP. (2017). Epidémiologie des malformations congénitales visible à la naissance à Yaoundé. Health Sci. Dis, 18 (4): 53-62.

10. Konaté-Lankoandé, C T. (2008). Malformations congénitales externes des nouveau-nés dans les services de Gynécologie et d'Obstétrique du CHU-YO et de Chirurgie du CHU-CDG.A propos de 49 cas. Thèse de Médecine/Université de Ouagadougou; No 3, 99p. 
11. Lubala, TK. (2011). Etude des malformations congénitales cliniquement visibles à la naissance Lubumbashi, p.4, [En ligne].http://www.memoireonline.com. [Consulté le 13/01/19].

12. Mouafo, TF, Mohe, N, Diath, AG. (2004). Malformations ano-rectales dans le service de chirurgie pédiatrique du CHU de Yopougon. Abidjan, Cote d'Ivoire. Mali médical, 35-38.

13. Nazer, HJ, Cifuentes, OL, Aguila, RA, Ureta, LP, Bello, PMP, Correa, CF, Melibosky, RF. (2005). Edad materna y malformaciones congénitas: un registro de 35 años. 1970-2005 [L'association entre l'âge maternel et les malformations congénitales]. Révérend Med Chil., 135(11):1463-9.

14. Organisation Mondiale de la Santé: Anomalies congénitales. A63/10. Avril 2010. [En ligne] http://apps.who.int/gb/ ebwha/pdf_files/ WHA63/A63_10-fr.pdf. [Consulté le 10/01/19].

15. Sabiri, N, Kabiri, M, Razine, R, Kharbach, A, Berrada R, Barkat, A. (2013). Facteurs de risque des malformations congénitales: étude prospective à la maternité Souissi de Rabat au Maroc. Journal de pédiatrie et de puériculture, 26 (4) : 198-203.

16. Shawky, RM, Sadik, DI. (2011). Congenital malformations prevalent among Egyptian children and associated risk factors. The Egyptian Journal of Medical Human Genetics, 12 (1): 69-78. https://doi.org/10.1016/j.ejmhg.2011.02.016

17. Youl, H.(2011). Malformations congénitales reconnaissables à la naissance chez les nouveau-nés dans le département de pédiatrie du CHU SOURO SANOU. Université de Ouagadougou. Thèse de médecine. [En ligne]. http://www.beep.ird.fr/collect/uouaga/index/assoc/ M12613.dir/M12613.pdf [Consulté le 10/02/19] 